\title{
From Correlation to Causality: Microarrays, Cancer, and Cancer Treatment
}

\author{
Geraldine M. O'Neill ${ }^{1}$, Daniel R. Catchpoole ${ }^{1}$, and Erica A. Golemis ${ }^{2}$ \\ ${ }^{1}$ Oncology Research Unit, The Children's Hospital at Westmead, Westmead, Australia, and ${ }^{2}$ Division of Basic \\ Science, Fox Chase Cancer Center, Philadelphia, PA, USA
}

BioTechniques 34:S64-S71 (March 2003)

\section{INTRODUCTION}

The clinical treatment of cancer is in the process of a great leap forward, with the use of microarray-based technologies holding the promise to derive previously unobtainable insights into many aspects of disease pathogenesis. In surveying the past five years of progress, it is apparent that use of microarrays to query tumor samples and matching model systems can provide useful information concerning tumor classification, prognosis, and response to drug treatment. Further uses of microarrays in cancer biology have been proposed, with particular interest in prospects of this technology to elucidate cancer etiology and identify critical signal transduction pathways relevant to cancer drug development. In this review, we summarize the status of these fields and use insights gleaned from the extensive use of microarrays in studies of gene function versus gene expression profiles in model organisms such as yeast to identify critical issues for ongoing application development.

\section{CURRENT AND DEVELOPING APPLICATIONS}

From its invention, the potential of microarray-based transcriptional profiling to elucidate highly complex biological systems has been apparent. Because of its multifactorial nature, its long disease course, and the relative inability to access early-stage transformed populations, cancer is without question one of the most complex diseases associated with a significant morbidity and mortality. Hence, much effort has been devoted to adapting the use of microarrays to different aspects of cancer research and treatment.

At present, there are some highly validated uses for microarrays in clinical study and treatment of cancer. Of these, an early milestone was the work of Khan et al., in which hierarchical algorithms were used to distinguish rhabdomyosarcoma-specific gene profiles that could be used for diagnostic purposes (1). Soon after, Golub and colleagues (2) successfully applied microarrays to the problems of identification of new cancer classes (class discovery) and the assignment of tumors to known classes (class prediction). In a blinded study, through use of class pre- diction algorithms, they first demonstrated that it was possible to distinguish between acute myeloid leukemia (AML) and acute lymphoblastic leukemia (ALL) solely on the basis of transcriptional profile. Subsequent work by a number of groups addressed the identification of disease specific profiles for diffuse large B-cell lymphoma (DLBCL) (3), breast tumors (4), and small round blue cell tumors (SRBCTs) (5), among others.

In each case, application of a variety of data analysis techniques to develop signature mRNA profiles for tumors that are difficult to separate by conventional histopathological microscopic examination revealed the identification of subclasses suggestive of different cellular origins of tumors, and in many cases allowed better prediction of the disease prognosis. Some recent studies have emphasized this latter application, with studies by Yeoh et al. on pediatric ALL (6), Rosenwald et al. on DLBCL (7), and van't Veer et al. on breast cancer (8), focusing on risk stratification of patients, to predict survival outcomes following therapy. An ultimate goal of this work is to provide the intellectual basis for patient-specific, "personalized" medical treatment.

In general, one highly desirable future use of microarrays would be to provide guidance for the development and utilization of chemotherapeutic drugs. This is an area of active investigation. Some recent studies utilize a number of different approaches, including the creation of transcriptional profiles in yeast $(9,10)$, cell lines $(11-13)$, or xenografts (14) following treatment with commonly used chemotherapeutic agents, to establish a profile to allow the typing of novel agents of interest. A second theme has been to emphasize the use of profiles to gain insight into the mode-of-action and molecular targets of new, purportedly "pathway-targeted" drugs (e.g., 10,12,15,16). Providing a bridge between the analysis of cancer development, and analysis of the mode of drug action, some researchers have begun to apply microarrays to the classification of treatment-induced acute myeloid leukemias (17).

Finally, a point of keen interest in the cancer field would be the use of microarrays to identify the initiating signaling events that lead to the induction of cancer. Many researchers have begun to compare profiles found in normal cells, with matched cancers of different degrees of malignancy, to try to identify the molecular mechanisms associated with carcinogenesis (e.g., 
18,19). Others have contrasted the transcriptional profiles found in cancers of different types to identify systematic changes and consistencies associated with cancer development (20); an alternative approach has been to establish profiles of gene expression change associated with the controlled induction of an oncogene or tumor suppressor, such as p53 (21). The relative fruitfulness of these diverse approaches will become clear over the next decade.

\section{CRITICAL ISSUES FOR DEVELOPING APPLICATIONS: MOVING FROM mRNA PROFILES TO SIGNAL TRANSDUCTION}

For applications such as tumor identification and risk stratification, the use of microarray data is clear: to provide a diagnostic pattern that can serve as a basis for treatment decisions and patient counseling. Similarly, although much systematic work remains to be done to develop databases containing requisite information (for example, combining patient genotype, medical history, array profile, etc.), there are few theoretical impediments to the ultimate use of microarray data to create patient-specific treatment regimens. For these clinical purposes, the set of genes included in the diagnostic pattern is not an issue, as long as the gene profile is reliably associated with a given outcome.

In contrast, for basic science investigators or drug developers, there are more issues at play in assessing the use of microarray data. Over the past 20 years, a view of cancer has developed in which signaling changes affecting multiple categories of cellular function together cooperate to create a transformed phenotype. These changes promote evasion of apoptosis; independence of positive or negative exogenous growth regulatory factors; stimulation of tissue invasion and metastasis; delimited replication, and angiogenesis (22). In addition, given the variability in response of tumor classes to chemotherapy, an additional set of changes observed in some but not all tumors bear on drug resistance. To use microarrays to better understand the molecular basis of cancer development, and thus to identify critical control points against which pharmaceutical agents can be targeted, requires a number of assumptions. For example, it must be assumed that changes in mRNA levels and proteins for genes in these classes occur in tumors, are detectable by microarray technology, and are associated directly with a causal role in the cancers in which they are observed. Data bearing on these assumptions are discussed below.

\section{How Complete is the Data Set?}

A constant issue in expression profiling experiments is the issue of sensitivity. A notable advantage of microarrays over proteomic assays is the fact that PCR-based approaches expand the range of detectable genes. Current technologies used for microarrays tend to work best with genes expressed at 5-10 copies/cell or higher. This poses a potential problem, as studies of yeast (23,24 and references therein) indicate that copy numbers of individual mRNAs vary over 6 orders of magnitude, and that $75 \%$ of genes are expressed at less than 1 copy/cell. Further, many functionally critical transcription factors are present at as low as 1/1000 copies/cell under conditions of repression and only 1-2 copies/cell under induced conditions. Similar low abundance of some critical mammalian signaling proteins has been reported. In contrast, genes associated with general homeostasis functions such as metabolism, protein synthesis, and cytoskeletal structures are more highly expressed, and dominate expression profiles. Given issues related to scalability of signal and detection limits, it may be that the most critical signaling changes are currently at the lower limit of detection or beyond, although current technological efforts are attempting to address this problem (e.g., 25). Finally, it is clear that much genomic complexity is derived from the expression of isoforms (26) that can exhibit unique, nonredundant functions. However, few microarrays currently distinguish between the many multi-isoform families present in the genome.

A related issue is the question of population accessibility, for identification of critical causative changes. It is well-known that cancer is a multistep process (27). As described above, following the development of a "mature" cancer that has achieved sufficient mass to be diagnosed, the resulting tumor maintains a profile that can be used for diagnostic studies that reflects the developmental origin of the tumor, but contains multiple changes related to cancer progression. This complicates the analysis of the significance of any given change as causative of a tumor, particularly as a large fraction of the mRNA profile changes found in tumors simply reflect the fact that aggressively cancerous cells tend to be in active cell cycle, and hence express genes known to be expressed in cycling cells $(28,29)$. Conversely, as tumors are detected at earlier stages, it becomes more difficult to identify a pure tumor population, well separated from surrounding matrix and stroma. Laser capture microdissection and advances in the analysis of gene expression changes in single cells (e.g., using marked model cell systems) are currently attempting to address the issue of early cancerous changes, more closely linking changes in gene expression to etiology (30-32).

\section{Do Changes in Transcript Level Reflect Changes in Protein Level?}

Within cells, signaling is accomplished largely through the action of proteins: hence, an essential question is whether changes in mRNA result in commensurate changes in the expression of the proteins they encode. In fact, this may not be the case. Based on extensive work comparing transcriptional and proteomic signatures in yeast $(23,33,34)$, it has been determined that large changes in mRNA levels are generally paired with much lower levels of change in protein levels [e.g., mRNA:protein fold change ratio of 490:10 for GAL2, 162:6.9 for GAL1, 55:11 for GAL10 following galactose induction (15)]. Small changes in mRNA levels (e.g., 3- to 5-fold) are less predictable, with matching protein levels sometimes varying similarly, but frequently to a lesser degree in the same direction, or in some cases in the opposite direction (33). Such results clearly emphasize the importance of posttranscriptional control mechanisms in determining protein levels.

\section{What is the Correlation Between Functionality (Causality) and Transcriptional Change in a Given Process?}

In this context, and given the current knowledge of signal 
transduction networks, it is not clear that changes in transcript levels or protein levels play the most important role in cancer development. Discussions of critical changes in cell growth controls relevant to oncogenic transformation to date have tended to emphasize changes in properties of protein-protein association and posttranslational modification, leading to altered protein activity, rather than gross changes in protein levels [e.g., see discussion of factors contributing to Ras-dependent transformation (35)]. Establishment of disease causality for an observed transcriptional change is a nontrivial problem.

There is no question that it has been possible in some cases to inspect a transcriptional profile characteristic of a type of cancer, select genes with previously defined interesting biology, and demonstrate that changes in the function of that gene can affect cancer-relevant properties of cells. Examples of such intelligent gene selection include the identification of RhoC (36) and PRL3 (37) as promoters of metastasis, based on their transcriptional up-regulation in melanomas and colorectal cancer, respectively. Similarly, analysis of the profile of the genes induced following activation of the oncoprotein Raf (38) included many genes with functions relevant to cancerous transformation. However, the vast majority of genes still lack assigned function, limiting for at least the next several years the ability to select critical targets based on prior knowledge. In this context, an important question is whether it is reasonable to assume that, because a gene undergoes a significant change in mRNA levels following a stimulus, the protein encoded by that gene plays a role in response to that stimulus.

In some cases, this may be a valid assumption. For example, in an early report demonstrating the value of microarrays for pathway discovery, it was possible to tease apart the contribution of different mitogen-activated protein kinase (MAPK) signaling modules to pheromone signaling in Saccaromyces cerevisiae (39). The authors demonstrated that each component downstream of the pheromone receptor (ste2) was required for pheromone-mediated gene expression, therefore concluding that the entire transcriptional response is derived from pathway dependent activation of Ste12p. An interesting observation by this group is that disrupting signal transmission at intermediate steps did not result in cross-talk activation of other MAPK pathways. Further, the authors were able to dissect the sequential activation of differentially regulated MAPK pathways during the complex multi-signaling pathway phenotype of mating projection formation.

However, such clear results are not necessarily the norm. As scientific resources have continued to develop in a post-genomic era, a particularly useful resource for analysis of the relation between gene expression change and gene function is the availability of arrayed yeast knockout banks, in which each open reading frame $(\mathrm{ORF})$ is individually deleted $(9,40,41)$. Several groups have now performed global function/transcription analyses. In this type of analysis, for a given stimulus, the requirement of each individual gene is assessed based on phenotype of the knockouts in the bank under conditions of stimulation: while in parallel, the transcriptional profile following the stimulus is assessed in a wild-type strain background. Conditions so far assessed include induction of DNA damage (by cisplatin, hydrogen peroxide, or radiation), or growth on medium incorporating high salt, sorbitol, galactose, high $\mathrm{pH}$, minimal nutrients, or the fungicide nystatin. In the best case correlation, it was deter- mined that $7 \%$ of the mRNAs whose transcripts changed following galactose induction had functions related to optimal growth on galactose, while for other stimuli (high salt, high $\mathrm{pH}$, sorbitol), overlaps of only $0.3 \%-3 \%$ were observed. Significantly, in assessment of response to DNA damaging agents, the majority of genes known to be essential for that response showed no significant variation in mRNA levels following damage induction, indicating that the steady state levels of these mRNAs and encoded proteins were sufficient to provide protection.

\section{Summary}

The preceding discussion indicates that expression changes for mRNA do not necessarily mimic protein expression changes; that genes expressed at low levels are frequently missed from analyses; that the populations of cells assessed in cancer frequently represent a late-stage, rather than early transformed population, and hence contain many nonspecific changes; that of the many changes in transcript levels observed after stimuli include genes with no clear functional requirement in the process being tested; and that posttranslational changes are critically important in mediating signaling. Eventually, the questions of data quality and target accessibility noted above are likely to be at least partly addressed through improvements in technology. However, the relationship of expression change to causality remains a daunting problem, which strongly suggests it will be challenging to use profile data to identify critical players for a given process based on candidate-picking from the up-regulated pool of genes in the absence of extensive pre-existing pathway information. In this context, then, what information can be harvested from study of profiles that is useful for gaining novel insights into critical pathways and protein targets in cancer?

\section{ISSUES OF SPECIFICITY: MICROARRAYS AND DRUG STUDIES}

Ideally, over the next decade the use of microarrays will assist in the understanding of the cellular mechanisms underlying drug response, and identify targets of novel pathway directed drugs. To accomplish these goals, it is necessary to first determine whether the mRNA profiles resulting from drug treatment of cells reflects, or can be made to yield, an interpretable pattern specific to a drug being assessed. Beyond the issues cited in the preceding section, there are a number of other factors that bear on analysis of specific drug response. As our understanding of cellular mechanisms of survival and apoptosis increases, it is becoming clear that most agents that were previously believed to have a single mode of action, in fact exert their cytotoxic (and cytostatic) effects through multiple cellular targets (42) likely to induce generic changes in transcriptional programs (also see Reference 43). Timing of assessment of transcriptional response after drug treatment is likely to be critical, for this and other reasons. Also, as is clear from the discussion of cancer-specific signatures provided above, different cancers are likely to have different "baseline" signatures of genes that may be relevant to drug response, raising the possibility of quite different apparent magnitudes of mRNA expression change dependent on cancer cell model used for a study. 
Several reports have indicated that these issues can be significant challenges, confounding easy interpretation of drug response data. Nevertheless, while confirming the problem, these early studies have begun to provide the basis for a solution. For example, in experiments performed in yeast (44-46), it has been shown that treatment of yeast with a diverse series of toxic chemicals or stressful environmental conditions induces a very large, common set of transcriptional changes. This set of changes, dubbed the environmental stress response (ESR) by one group, includes changes in the levels of genes involved in a diverse group of cellular defenses, including protein folding, protection against reactive oxygen species, control of energy storage, and DNA repair. Comparably, reference studies performed with different anticancer drugs against panels of human cancer cell line models (e.g., 11,13,20) have confirmed large groups of expression changes after the treatment with drugs which either contained genes associated with general stress response, or reflected the tissue of origin of the tumor. Nevertheless, and encouragingly, profiles of transcriptional response have been found to be nonidentical between drugs of different classes, and further, drugs with known similar modes of function tended to cluster more closely in analysis of induced profile change, than did drugs with distinct modes of function $(11,13)$.

These results indicate that with an appropriate algorithmic filter for general (ESR-like) genes, or with additional sources of functional information to guide analysis, useful insight into the mechanism of drug action might be gained. Members of this ESR set may, for example, account for a significant level of background in studies such as the Birrell and Giaever studies described above $(9,40)$. Further, it has been found that a considerable number of the ESR genes are induced by a specific and limited set of transcription factors (e.g., 46); as more such are identified, it will increasingly become possible to distinguish specific from general responses. Similarly, it will be ultimately possible to develop filters based on the background profile of gene expression in different cell lines standardly used for drug response analysis (e.g., the NCI-60 panel). Finally, other studies such as that of Cohen et al., providing insight into nontranscriptional controls leading to coexpression of genes (47) or the work of various teams defining genes whose expression tends to be hypervariable based on population variation (48) or for other reasons (49), will also contribute to the improvement of filters.

Another productive avenue has been to thoroughly integrate functional experiments with profiling approaches. Logically, if a drug is intended to target a specific cellular protein and eliminate or modulate its function, then cells treated with that drug will have a phenotype that is strongly related to that observed in cells deficient in the cognate target. While the profiles may not be identical, because a mutant represents a constitutive loss of function of a key protein through development and life of the cell, while drug treatment is more transient, still similarities should be significant. One straightforward, although still correlative, approach, similar to investigations into changes associated with cancer progression, is to perform analyses of closely related cell lines selected for gradually increasing resistance to a drug of interest (e.g., 50), on the theory that a consistent pattern of functionally relevant genes will appear against a backround of irrelevant variation. However, genotoxic effects may have a poten- tially confounding effect on the results, although this same criticism is true for all studies of model drug resistant cell lines (42). Moving from correlation to causation, an alternative strategy suggested by Marton et al. (16) has been to compare the mRNA profile in cells treated versus untreated with a protein-targeted drug: looking at both wild-type cells, and cells mutated for the drug target. In their examination of the transcriptional consequences of treatment with the drug FK506, again using a yeast model system, this group was able to clearly distinguish mRNA profile changes dependent on the drug effector protein calcineurin, hence linking outcome with stimulus.

More recently, Kuruvilla et al. extended such analysis to first screen a library of compounds to find those with the capacity to physically bind the yeast protein Ure $2 p$, then to sort chemicals with Ure2p-binding activity by microarray profiling in wildtype versus ure 2 mutant cells to find compounds that inhibited a subset of Ure2p-dependent transcripts (15). This approach should be widely generalizable for drug discovery efforts, particularly in cases where essential human genes have yeast or (potentially) Caenorhabditis elegans or Drosophila homologs. Another related approach with significant potential is to perform analysis of a drug of interest with an evolutionarily conserved target in both mammalian cells and in yeast, as has been recently done for the proteasome inhibitor PS-341 $(10,12)$. As recently adapted techniques such as the use of small interfering RNAs [siRNAs (51)] begin to render routine comparison of a drug with a gene-directed ablation in mammalian cells, the ease and applicability of these approaches will continue to increase.

Finally, it is possible to envisage use of microarrays in discovery efforts to identify new drug targets. One potentially useful avenue to this end is to derive hypotheses on the signaling functions of mammalian proteins based on the functions of homologous molecules in yeast or other model organisms. For example, among the uncharacterized ORFs studied by Hughes et al. (45) clone $E R G 28$ was shown to play a role in sterol biosynthesis. It was then further demonstrated that the human homologue of ERG28 could complement an ERG28 mutant yeast strain, suggesting that the human homologue may play a role in the human cholesterol biosynthetic pathway. Considering the large degree of conservation between signaling pathways in yeast and humans, it is likely that this approach, assigning function to the yeast molecule and then testing whether the human homologue can rescue the phenotype, may be broadly applicable (e.g., 52). Although at present, there remain a number of barriers to directly applying a microarray-based discovery approach to mammalian signal transduction pathways, improvements in technology and understanding of pathway connectivity may enable these approaches in the future.

\section{ISSUES OF SPECIFICITY: MICROARRAYS AND SIGNAL TRANSDUCTION}

An important lesson from the above discussion of critical issues for analysis of drug-cell interactions is that it is not necessary to identify a transcriptionally regulated drug target to generate useful information about the interaction of a drug with cellular pathways. It may be totally impossible to identify transcriptionally regulated targets with a critical and specific func- 
tion in a given pathway. Nevertheless, with an appropriate subtractive filter, and with tools to finely manipulate signaling pathways known in advance to be closely related, microarrays can provide a means to access one of the most challenging current problems in understanding cell growth control disturbance; that is, how signaling from multiple different upstream inputs is summed through the action of a conserved downstream effector machinery, and how this becomes disrupted following the constitutive activation of oncogenic signaling pathways in cancer. In this conceptual shift, the actual identity of regulated transcripts becomes less important, while it is the general pattern of overlap or divergence between profiles obtained that becomes important (see References 53-55).

Returning again to yeast to illustrate this point, it was earlier proposed that global transcriptional responses may represent a phenotypic fingerprint of particular signaling pathway (45). This proposal was tested by taking a "compendium approach" to signaling in yeast. A collection of 300 full-genome expression profiles was prepared in $S$. cerevisiae corresponding to mutations in both well-characterized genes and uncharacterized open reading frames, as well as treatments with compounds with known molecular targets, compared with wild-type or mock-treated profiles (45). Cluster analysis of these data sets led to generally coherent functional groupings of transcript profiles. In this case, there was a clear trend for related transcript profiles among different mutants that affected the same cellular process. Based on coregulation of known and novel transcripts, the researchers were able to gain new insights into the transcriptional target specificity of signaling proteins in the tested pathways, and to some extent were able to identify novel proteins with important functions (see below).

Some groups have begun to attempt similar compendium approaches to analyze signal transduction pathways in mammalian cell models relevant to cancer. A pair of examples illustrates the complexity of this type of analysis in higher eukaryotes. In a first experiment, DNA microarray analysis has been used to dissect and contrast signal transduction profiles from individual protein interaction motifs, within one molecule. Again, this experimental protocol required prior specialized knowledge of the structural and functional components of the protein analyzed. Specifically, mutant PDGF receptors were engineered in which various tyrosine-containing protein interaction sites were replaces with phenylalanine (56). Effectively, this should result in the uncoupling of a particular site on the receptor from its downstream signaling pathway. The results showed that sites on the receptor that would normally interfere with PDGF activation through variety of different effectors nevertheless induce broadly overlapping groups of transcriptional targets, since near- 
ly all of the mutants tested resulted in stimulation of a similar expression response but at a reduced level. This can be taken to indicate that diverse pathways exert overlapping effects on immediate early gene targets. In contrast, subsequent studies by the authors contrasting the profiles following from different complements of ErbB receptors indicated a partially overlapping, but nevertheless quite distinct profile of gene response (57). The reasons these results differ from those in the prior study may bear on specific differences between PDGF and ErbB signaling, or differences in the cell types assayed, or other considerations, remain to be resolved, and are questions of considerable interest (for further discussion of relevant issues, see References 53-55).

Given that a major limitation of gene expression profiling application to the study of signal transduction pathways is the absence of information regarding protein expression, a number of researchers are attempting to combine expression profiling data with data from large scale protein-protein interaction screens. In one example, gene expression data was used to rank candidate pathways of interacting proteins in yeast $(58)$. The success of this "Netsearch" program was confirmed by the ability to accurately predict known $S$. cerevisiae MAPK signaling pathways, among others. This approach appears to have real potential in the modeling of signal transduction pathways in mammalian cells. There is much concerted effort directed at mapping mammalian protein-protein interaction networks and current advances in technology are greatly enhancing these efforts. Accessibility of these networks, such as those provided by Science in the Signal Transduction Knowledge Environment (http://stke. sciencemag.org), will facilitate the application of gene expression profiling data to interaction pathways in mammalian cells. While this approach still has some obvious limitations, including assumptions about the relationship between gene expression and protein levels, it is likely to provide an exponential increase in our understanding of mammalian signal transduction pathways. Knowledge of signal transduction pathways in normal cells will greatly aid in our ability to understand causal changes in transformed cells.

\section{CONCLUSION}

It is clear that microarrays have a significant contribution to make to studies of cancer. Some areas of application, such as cancer classification, are amenable to relatively straightforward analyses in the near future. Other areas, such as dissection of cancer-specific signaling and drug response, will require the development of a battery of approaches to exactly manipulate signaling pathways (e.g., 59,60), supported by extensive database resources and bioinformatic algorithm development, but ultimately have the potential to open entirely new areas of research.

\section{REFERENCES}

1.Khan, J., R. Simon, M. Bittner, Y. Chen, S.B. Leighton, T. Pohida, P.D. Smith, Y. Jiang, et al. 1998. Gene expression profiling of alveolar rhabdomyosarcoma with cDNA microarrays. Cancer Res. 58:5009-5013.

2.Golub, T.R., A. Goga, G.F. Barker, D.E.H. Afar, J. McLaughlin, S.K. Bohlander, J.D. Rowley, O.N. Witte, and D.G. Gilliland. 1996. Oligomerization of the ABL tyrosine kinase by the Ets protein TEL in human leukemia. Mol. Cell. Biol. 16:4107-4116.
3.Alizadeh, A.A., M.B. Eisen, R.E. Davis, C. Ma, I.S. Lossos, A. Rosenwald, J.C. Boldrick, H. Sabet, et al. 2000. Distinct types of diffuse large B-cell lymphoma identified by gene expression profiling. Nature 403:503-511.

4.Perou, C.M., T. Sorlie, M.B. Eisen, M. van de Rijn, S.S. Jeffrey, C.A. Rees, J.R. Pollack, D.T. Ross, et al. 2000. Molecular portraits of human breast tumours. Nature 406:747-752.

5.Khan, J., J.S. Wei, M. Ringner, L.H. Saal, M. Ladanyi, F. Westermann, F. Berthold, M. Schwab, et al. 2001. Classification and diagnostic prediction of cancers using gene expression profiling and artificial neural networks. Nat. Med. 7:673-679.

6.Yeoh, E.J., M.E. Ross, S.A. Shurtleff, W.K. Williams, D. Patel, R. Mahfouz, F.G. Behm, S.C. Raimondi, et al. 2002. Classification, subtype discovery, and prediction of outcome in pediatric acute lymphoblastic leukemia by gene expression profiling. Cancer Cell 1:133-143.

7.Rosenwald, A., G. Wright, W.C. Chan, J.M. Connors, E. Campo, R.I. Fisher, R.D. Gascoyne, H.K. Muller-Hermelink, et al. 2002. The use of molecular profiling to predict survival after chemotherapy for diffuse large-B-cell lymphoma. N. Engl. J. Med. 346:1937-1947.

8.van't Veer, L.J., H. Dai, M.J. van de Vijver, Y.D. He, A.A. Hart, M. Mao, H.L. Peterse, K. van der Kooy, et al. 2002. Gene expression profiling predicts clinical outcome of breast cancer. Nature 415:530-536.

9.Birrell, G.W., J.A. Brown, H.I. Wu, G. Giaever, A.M. Chu, R.W. Davis, and J.M. Brown. 2002. Transcriptional response of Saccharomyces cerevisiae to DNA-damaging agents does not identify the genes that protect against these agents. Proc. Natl. Acad. Sci. USA 99:8778-8783.

10.Fleming, J.A., E.S. Lightcap, S. Sadis, V. Thoroddsen, C.E. Bulawa, and R.K. Blackman. 2002. Complementary whole-genome technologies revea the cellular response to proteasome inhibition by PS-341. Proc. Natl. Acad. Sci. USA 99:1461-1466.

11.Dan, S., T. Tsunoda, O. Kitahara, R. Yanagawa, H. Zembutsu, T. Katagiri, K. Yamazaki, Y. Nakamura, and T. Yamori. 2002. An integrated database of chemosensitivity to 55 anticancer drugs and gene expression profiles of 39 human cancer cell lines. Cancer Res. 62:1139-1147.

12.Mitsiades, N., C.S. Mitsiades, V. Poulaki, D. Chauhan, G. Fanourakis, X Gu, C. Bailey, M. Joseph, et al. 2002. Molecular sequelae of proteasome in hibition in human multiple myeloma cells. Proc. Natl. Acad. Sci. USA 99:14374-14379

13.Scherf, U., D.T. Ross, M. Waltham, L.H. Smith, J.K. Lee, L. Tanabe, K.W Kohn, W.C. Reinhold, et al. 2000. A gene expression database for the molecular pharmacology of cancer. Nat. Genet. 24:236-244.

14.Zembutsu, H., Y. Ohnishi, T. Tsunoda, Y. Furukawa, T. Katagiri, Y. Ueyama, N. Tamaoki, T. Nomura, et al. 2002. Genome-wide cDNA microarray screening to correlate gene expression profiles with sensitivity of 85 human cancer xenografts to anticancer drugs. Cancer Res. 62:518-527.

15.Kuruvilla, F.G., A.F. Shamji, S.M. Sternson, P.J. Hergenrother, and S.L. Schreiber. 2002. Dissecting glucose signalling with diversity-oriented synthesis and small-molecule microarrays. Nature 416:653-657.

16.Marton, M.J., J.L. DeRisi, H.A. Bennett, V.R. Iyer, M.R. Meyer, C.J Roberts, R. Stoughton, J. Burchard, et al. 1998. Drug target validation and identification of secondary drug target effects using DNA microarrays. Nat. Med. 4:1293-1301.

17.Qian, Z., A.A. Fernald, L.A. Godley, R.A. Larson, and M.M. Le Beau. 2002. Expression profiling of CD34+ hematopoietic stem/progenitor cells reveals distinct subtypes of therapy-related acute myeloid leukemia. Proc Natl. Acad. Sci. USA 99:14925-14930.

18.Calvo, A., N. Xiao, J. Kang, C.J. Best, I. Leiva, M.R. Emmert-Buck, C. Jorcyk, and J.E. Green. 2002. Alterations in gene expression profiles during prostate cancer progression: functional correlations to tumorigenicity and down-regulation of selenoprotein-P in mouse and human tumors. Cancer Res. 62:5325-5335.

19.Ono, K., T. Tanaka, T. Tsunoda, O. Kitahara, C. Kihara, A. Okamoto, K. Ochiai, T. Takagi, and Y. Nakamura. 2000. Identification by cDNA microarray of genes involved in ovarian carcinogenesis. Cancer Res. 60:50075011.

20.Ross, D.T., U. Scherf, M.B. Eisen, C.M. Perou, C. Rees, P. Spellman, V. Iyer, S.S. Jeffrey, et al. 2000. Systematic variation in gene expression patterns in human cancer cell lines. Nat. Genet. 24:227-235.

21.Yoon, H., S. Liyanarachchi, F.A. Wright, R. Davuluri, J.C. Lockman, A. De La Chapelle, and N.S. Pellegata. 2002. Gene expression profiling of isogenic cells with different TP53 gene dosage reveals numerous genes that are affected by TP53 dosage and identifies CSPG 2 as a direct target of p53. Proc. Natl. Acad. Sci. USA 99:15632-15637. 
22.Hanahan, D. and R.A. Weinberg. 2000. The hallmarks of cancer. Cell 100:57-70.

23.Gygi, S.P., Y. Rochon, B.R. Franza, and R. Aebersold. 1999. Correlation between protein and mRNA abundance in yeast. Mol. Cell. Biol. 19:17201730 .

24.Holland, M.J. 2002. Transcript abundance in yeast varies over six orders of magnitude. J. Biol. Chem. 277:14363-14366.

25.Dudley, A.M., J. Aach, M.A. Steffen, and G.M. Church. 2002. Measuring absolute expression with microarrays with a calibrated reference sample and an extended signal intensity range. Proc. Natl. Acad. Sci. USA 99:75547559 .

26.Gunning, P. Protein Isoforms and Isozymes. In D.N. Cooper (Ed.), Encyclopedia of the Human Genome. Macmillan Reference Ltd., Nature Publishing Group, London. (In press.)

27.Vogelstein, B. and K.W. Kinzler. 1993. The multistep nature of cancer. Trends Genet. 9:138-141.

28.Cho, R.J., M. Huang, M.J. Campbell, H. Dong, L. Steinmetz, L. Sapinoso, G. Hampton, S.J. Elledge, et al. 2001. Transcriptional regulation and function during the human cell cycle. Nat. Genet. 27:48-54.

29.Whitfield, M.L., G. Sherlock, A.J. Saldanha, J.I. Murray, C.A. Ball, K.E. Alexander, J.C. Matese, C.M. Perou, et al. 2002. Identification of genes periodically expressed in the human cell cycle and their expression in tumors. Mol. Biol. Cell 13:1977-2000.

30.Iscove, N.N., M. Barbara, M. Gu, M. Gibson, C. Modi, and N. Winegarden. 2002. Representation is faithfully preserved in global cDNA amplified exponentially from sub-picogram quantities of mRNA. Nat. Biotechnol. 20:940-943.

31.Levsky, J.M., S.M. Shenoy, R.C. Pezo, and R.H. Singer. 2002. Single-cell gene expression profiling. Science 297:836-840.

32.Wang, W., J.B. Wyckoff, V.C. Frohlich, Y. Oleynikov, S. Huttelmaier, J. Zavadil, L. Cermak, E.P. Bottinger, et al. 2002. Single cell behavior in metastatic primary mammary tumors correlated with gene expression patterns revealed by molecular profiling. Cancer Res. 62:6278-6288.

33.Griffin, T.J., S.P. Gygi, T. Ideker, B. Rist, J. Eng, L. Hood,and R. Aebersold. 2002. Complementary profiling of gene expression at the transcriptome and proteome levels in Saccharomyces cerevisiae. Mol. Cell Proteomics 1:323-333.

34.Ideker, T., V. Thorsson, J.A. Ranish, R. Christmas, J. Buhler, J.K. Eng, R. Bumgarner, D.R. Goodlett, et al. 2001. Integrated genomic and proteomic analyses of a systematically perturbed metabolic network. Science 292:929934

35.Kolch, W. 2000. Meaningful relationships: the regulation of the Ras/Raf/ MEK/ERK pathway by protein interactions. Biochem. J. 351:289-305.

36.Clark, E.A., T.R. Golub, E.S. Lander, and R.O. Hynes. 2000. Genomic analysis of metastasis reveals an essential role for RhoC. Nature 406:532-535.

37.Saha, S., A. Bardelli, P. Buckhaults, V.E. Velculescu, C. Rago, B. St Croix, K.E. Romans, M.A. Choti, et al. 2001. A phosphatase associated with metastasis of colorectal cancer. Science 294:1343-1346.

38.Schulze, A., K. Lehmann, H.B. Jefferies, M. McMahon, and J. Downward. 2001. Analysis of the transcriptional program induced by Raf in epithelial cells. Genes Dev. 15:981-994.

39.Roberts, C.J., B. Nelson, M.J. Marton, R. Stoughton, M.R. Meyer, H.A. Bennett, Y.D. He, H. Dai, et al. 2000. Signaling and circuitry of multiple MAPK pathways revealed by a matrix of global gene expression profiles. Science 287:873-880.

40.Giaever, G., A.M. Chu, L. Ni, C. Connelly, L. Riles, S. Veronneau, S. Dow, A. Lucau-Danila, et al. 2002. Functional profiling of the Saccharomyces cerevisiae genome. Nature 418:387-391.

41.Winzeler, E.A., D.D. Shoemaker, A. Astromoff, H. Liang, K. Anderson, B. Andre, R. Bangham, R. Benito, et al. 1999. Functional characterization of the $S$. cerevisiae genome by gene deletion and parallel analysis. Science 285:901-906.

42.Johnstone, R.W., A.A. Ruefli, and S.W. Lowe. 2002. Apoptosis: a link between cancer genetics and chemotherapy. Cell 108:153-164.

43.Lam, L.T., O.K. Pickeral, A.C. Peng, A. Rosenwald, E.M. Hurt, J.M. Giltnane, L.M. Averett, H. Zhao, et al. 2001. Genomic-scale measurement of mRNA turnover and the mechanisms of action of the anti-cancer drug flavopiridol. Genome Biol. 2:RESEARCH0041.

44.Gasch, A.P., P.T. Spellman, C.M. Kao, O. Carmel-Harel, M.B. Eisen, G. Storz, D. Botstein, and P.O. Brown. 2000. Genomic expression programs in the response of yeast cells to environmental changes. Mol. Biol. Cell 11:4241-4257.
45.Hughes, T.R., M.J. Marton, A.R. Jones, C.J. Roberts, R. Stoughton, C.D. Armour, H.A. Bennett, E. Coffey, et al. 2000. Functional discovery via a compendium of expression profiles. Cell 102:109-126.

46.Jelinsky, S.A., P. Estep, G.M. Church, and L.D. Samson. 2000. Regulatory networks revealed by transcriptional profiling of damaged Saccharomyces cerevisiae cells: Rpn4 links base excision repair with proteasomes. Mol. Cell. Biol. 20:8157-8167.

47.Cohen, B.A., R.D. Mitra, J.D. Hughes, and G.M. Church. 2000. A computational analysis of whole-genome expression data reveals chromosomal domains of gene expression. Nat. Genet. 26:183-186.

48.Oleksiak, M.F., G.A. Churchill, and D.L. Crawford. 2002. Variation in gene expression within and among natural populations. Nat. Genet. 32:261266.

49.Pritchard, C.C., L. Hsu, J. Delrow, and P.S. Nelson. 2001. Project normal: defining normal variance in mouse gene expression. Proc. Natl. Acad. Sci. USA 98:13266-13271.

50.Turton, N.J., D.J. Judah, J. Riley, R. Davies, D. Lipson, J.A. Styles, A.G. Smith, and T.W. Gant. 2001. Gene expression and amplification in breast carcinoma cells with intrinsic and acquired doxorubicin resistance. Oncogene 20:1300-1306.

51.Tuschl, T., P.D. Zamore, R. Lehmann, D.P. Bartel, and P.A. Sharp. 1999. Targeted mRNA degradation by double-stranded RNA in vitro. Genes Dev. 13:3191-3197.

52.Steinmetz, L.M., C. Scharfe, A.M. Deutschbauer, D. Mokranjac, Z.S. Herman, T. Jones, A.M. Chu, G. Giaever, et al. 2002. Systematic screen for human disease genes in yeast. Nat. Genet. 31:400-404.

53.Boutros, M., H. Agaisse, and N. Perrimon. 2002. Sequential activation of signaling pathways during innate immune responses in Drosophila. Dev. Cell 3:711-722.

54.Shaffer, A.L., A. Rosenwald, E.M. Hurt, J.M. Giltnane, L.T. Lam, O.K Pickeral, and L.M. Staudt. 2001. Signatures of the immune response. Immunity 15:375-385.

55.Vaudry, D., P.J. Stork, P. Lazarovici, and L.E. Eiden. 2002. Signaling pathways for PC12 cell differentiation: making the right connections. Science 296:1648-1649.

56.Fambrough, D., K. McClure, A. Kazlauskas, and E.S. Lander. 1999. Diverse signaling pathways activated by growth factor receptors induce broadly overlapping, rather than independent, sets of genes. Cell 97:727-741.

57.Sweeney, C., D. Fambrough, C. Huard, A.J. Diamonti, E.S. Lander, L.C. Cantley, and K.L. Carraway, III. 2001. Growth factor-specific signaling pathway stimulation and gene expression mediated by ErbB receptors. J. Biol. Chem. 276:22685-22698.

58.Steffen, M.A., A.A. Petti, J. Aach, P. D’Haeseleer, and G.M. Church. 2002. Automated modelling of signal transduction networks. BMC Bioinformatics 3:34-45.

59.Harris, K., R.E. Lamson, B. Nelson, T.R. Hughes, M.J. Marton, C.J Roberts, C. Boone, and P.M. Pryciak. 2001. Role of scaffolds in MAP kinase pathway specificity revealed by custom design of pathway-dedicated signaling proteins. Curr. Biol. 11:1815-1824.

60.Serebriiskii, I.G., O. Mitina, E. Pugacheva, E. Benevolenskaya, E. Kotova, G.G. Toby, V. Khazak, W.G. Kaelin, et al. 2002. Detection of peptides, proteins, and drugs that selectively interact with protein targets. Genome Res. 12:1785-1791.

\section{Address correspondence to:}

Dr. Erica A. Golemis

Fox Chase Cancer Center

7701 Burholme Avenue

Philadelphia, PA 19111, USA

e-mail:EA_Golemis@fccc.edu 